PROTEIN FOLDING

\title{
Sweetening the deal
}

Science $\mathbf{3 4 0}, \mathbf{9 7 8 - 9 8 1 ( 2 0 1 3 )}$

Protein folding in the endoplasmic reticulum can be assisted by chaperones, but this process is energetically costly. Xu et al. suspected that there should be a mechanism by which protein folding is terminated to avoid wasting energy in futile refolding cycles. To investigate this possibility, the authors used several variants of GFP to explore the impact of folding rate on folding success. The authors observed that GFP localized in the endoplasmic reticulum of wild-type cells folds very poorly. However, a fast-folding GFP was able to fold successfully. The authors speculated that protein mannosylation might serve as a covalent modification marking proteins for folding termination. Indeed, dol-P-Man:protein O-mannosyltransferases (Pmts) are known to mannosylate folding-defective proteins in the endoplasmic reticulum, but the purpose of this modification was unclear. Purification of the two GFP constructs using a concanavalin A (ConA) affinity column to separate glycosylated and nonglycosylated proteins revealed that the fraction of slow-folding GFP bound to the column was larger than the fraction of fast-folding GFP. However, deletion of the yeast mannosyltransferases Pmt1 and Pmt2 reduced or abolished binding of the slow-folding GFP to ConA in single and double mutants, respectively. The double mutant also showed increased binding of the chaperone Kar2 to another folding-defective GFP construct. These data support a model where Pmt-mediated protein mannosylation provides a new mechanism of quality control by directly terminating unproductive folding.

tRNA MODIFICATIONS

\section{A structured answer}

Nature, published online 15 May 2013; doi:10.1038/nature12180

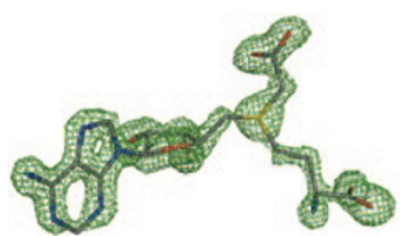

Post-transcriptional tRNA modifications have roles in tRNA stability and in ribosomal decoding. For instance, Gramnegative bacteria frequently contain 5-oxyacetyl uridine ( $\mathrm{cmo5 \textrm {U }}$ ) bases in the anticodon wobble position to read four degenerate codons. cmo5U is derived from 5-hydroxyuridine (ho5U) in a process that is dependent on chorismate biosynthesis and $\mathrm{CmoA}$ and $\mathrm{CmoB}$, two enzymes in the $S$-adenosyl-L-methionine (SAM)-dependent methyltransferase superfamily. Kim et al. now identify a new SAM metabolite, carboxy-Sadenosyl-L-methionine (Cx-SAM) and show that it is made by $\mathrm{CmoA}$ and used by $\mathrm{CmoB}$ for the biosynthesis of cmo5U. An X-ray crystal structure of CmoA from Escherichia coli revealed electron density consistent with a modified SAM moiety. Isolation and LC/MS analysis identified the metabolite as CX-SAM. Biochemical characterization showed that CmoA catalyzes the conversion of SAM to $\mathrm{Cx}$-SAM and is dependent on prephenate, a chorismate metabolite. ${ }^{13} \mathrm{C}$ labeling showed that the carboxyl group of Cx-SAM is derived from carbon dioxide generated at the CmoA active site by decarboxylation of prephenate. Deuterium labeling of SAM revealed that prephenate also performs a substrate-assisted catalytic role by generating a general base required for formation of the stabilized carbanion that ultimately receives the carboxyl functionality. Finally, the authors demonstrated that $\mathrm{CmoB}$ uses $\mathrm{Cx}-\mathrm{SAM}$ to catalyze the in vitro carboxymethylation of ho5U-modified RNA. In addition to elucidating the biosynthesis of $\operatorname{cmo5U}$, the study outlines a structure-guided approach to metabolite discovery.

PROTEIN FOLDING

\section{Retaining clients}

Mol. Cell, published online 16 May 2013; doi:10.1016/j.molcel.2013.04.016

Quality control mechanisms in the early secretory pathway facilitate protein folding and oligomeric assembly. For example, ERp44 is an oxidoreductase that prevents orphan subunits of disulfide-linked oligomers from being secreted. However, it was unclear how cycling of ERp44 between the endoplasmic reticulum and cis-Golgi was related to thiol-mediated client retention. Vavassori et al. use 1-anilinonaphthalene-8sulfonate-binding fluorescence spectroscopy and a maleimide-based probe to show $\mathrm{pH}$-dependent accessibility of the reactive cysteine (Cys29) of ERp44 in vitro. In cells, if the normal decrease in $\mathrm{pH}$ in the early secretory pathway compared to the endoplasmic reticulum was neutralized, ERp44-client interactions, which are normally enhanced at this location, were inhibited. In silico analyses based on a previously published crystal structure predicted that Cys29 would be deprotonated and occluded by the C-terminal tail at neutral $\mathrm{pH}$ but protonated and accessible at low $\mathrm{pH}$. Mutation of the residues that putatively stabilize protonated Cys 29 abolished the $\mathrm{pH}$ sensitivity of ERp44-client interactions in vitro and rendered these interactions constitutive in cells. Taken together, these data support a model in which ERp44 engages clients in the early secretory pathway, shuttles them back to the endoplasmic reticulum and releases them, owing in part to a $\mathrm{pH}$-dependent conformational change leading to occlusion of Cys 29 by the C-terminal tail.

MICROBIOLOGY

\section{A lipid under stress \\ Science 340, 837-841 (2013)}

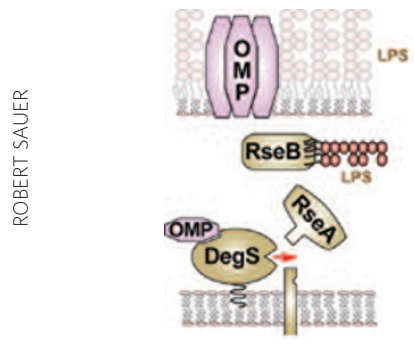

In Gram-negative bacteria, $\sigma^{\mathrm{E}}$ enhances transcription of genes whose products protect or restore the outer membrane after stressinduced damage. Periplasmic accumulation of outer-membrane proteins (OMPs) in E. coli activates degradation of RseA, a transmembrane protein and $\sigma^{\mathrm{E}}$ inhibitor, by the DegS protease. Inhibition of the RseAbinding partner RseB is also required to allow degradation and $\sigma^{\mathrm{E}}$ function, but the nature of the inhibiting signal is unknown. Lima et al. now show that lipopolysaccharide (LPS) and its variants can dissociate RseARseB complexes in vitro. Portions of LPS, including Lipid A fragments that contain GlcNAc sugars and N-linked acyl chains, also dissociate RseA-RseB. LPS fragments bind RseB directly and stabilize a tetrameric form that does not bind RseA. Using a partially functional LptD translocon, which inserts LPS into the outer membrane, the authors show that increased periplasmic LPS relieves RseB inhibition of RseA cleavage and activates the stress response. These results suggest that periplasmic buildup of the two major outer membrane components allows bacteria to sense and respond to stress: LPS frees the RseA inhibitor of $\sigma^{\mathrm{E}}$ to allow its degradation, and OMPs activate this degradation reaction.

Written by Mirella Bucci, Amy Donner, Joshua M. Finkelstein, Catherine Goodman \& Terry L. Sheppard 